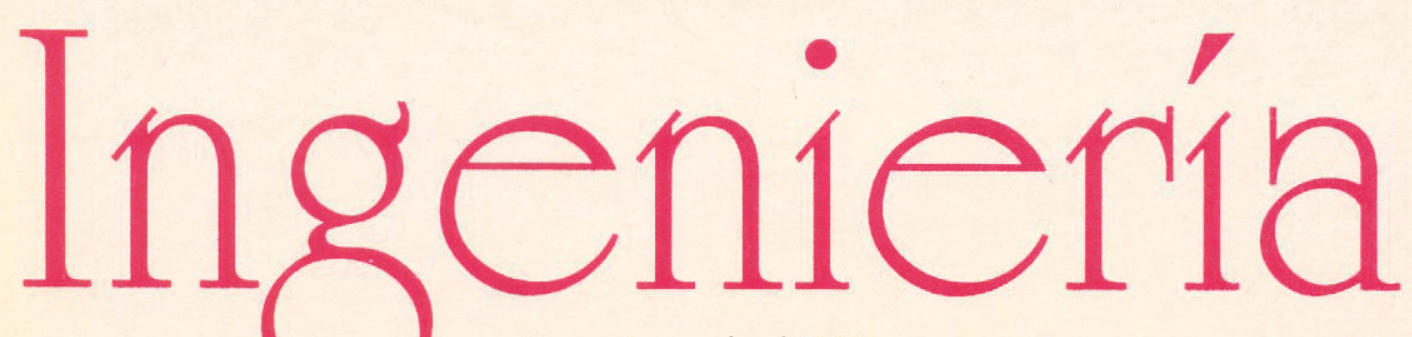

Revista de la Universidad de Costa Rica ENERO/JUNIO 1994 VOLUMEN 4 N 1

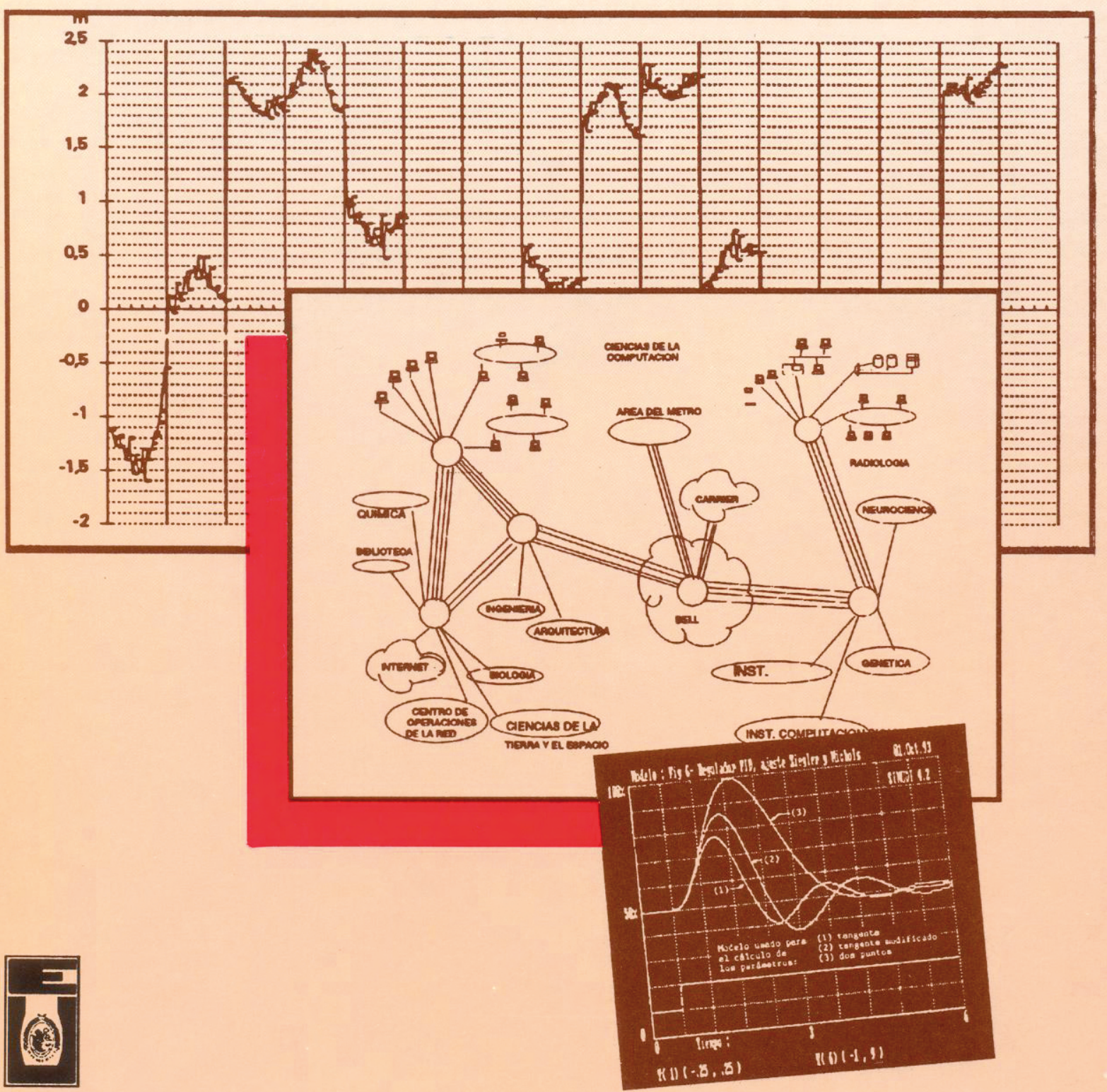




\section{DE LAS COPIAS PIRATAS DE PROGRAMAS DE COMPUTACIÓN AL DERECHO DEL CONOCIMIENTO}

\section{RESUMEN}

El problema de las copias de programas de computadoras-mal llamadas copias "piratas"- no se ha enfocado con perspectiva adecuada. Fuerzas interesadas en beneficios particulares han visto de forma limitada este asunto, proponiendo consideraciones legales y técnicas que podrían lesionar, severamente, la industria de la programación y de la informática en general.

Este artículo pretende enfocar la protección de programas ("software") desde una perspectiva más realista y libre de concepciones mercantiles o de corto plazo.

Se pretende dilucidar algunos puntos que no han sido considerados en la problemática o bien no tratados con la importancia debida. Además, se pretende informar cómo la legislación actual protege, en gran parte, los derechos del poseedor.

Por lo tanto existe solución al problema planteado. Se insiste en la necesidad de tratar este asunto considerando: la función que tienen los programas de computadoras enla industria informática a nivel mundial Ytambién la contribución de esta a su desarrollo.

\section{SUMMARY}

The problem of copying of computer programs - called «software piracy»- has not been approached from an adequated point of view. Groups -interested in particular benefits- have dealt with itvery limitely; making proposals of legal and technical aspects which could severely damage the prograrnming industry in particular and the informatics industry in global.

This paper deals with program protection (software) from a more realistic approach and free of marketing or short time considerations.

It pretends to Cla.ruy sorne aspects never take into account with respect to it or never covered with its real importance.

In addition, itpretends to give sorne lights ofhowthe actuallaws can protect the rights whoever has them and therefore, there are solutions to the given problerns. It insists in the need to focus this matter considering the function of computer programs as part of informatics industry and its contribution to the developing ofthe world.

Palabras dave: Programas, protección, conocimiento, derecho, desarrollo.

\section{1.- INTRODUCCIÓN}

Desde la aparición de las primeras computadoras de programa almacenado alrededor de los años cincuenta, se detectó la importancia de estos para el desarrollo de las computadoras y de la industria informática en general.

Compartir programas entre computadores similares sería de enorme beneficio. No existió la más mínima duda al respecto desde los inicios de la computación. Sin embargo, la poca similitud de estos en ese entonces, imposiblitó que se manifestaran tanto la necesidad como la posibilidad de copia de los programas. Tales manifestaciones

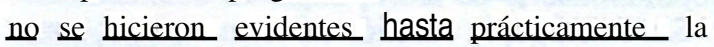

introducción de microcomputadores y la expansión de los computadores a todas las actividades humanas.

y como sucede con todo producto humano de interés general, también aparecieron aquellos que vieron la posibilidad de aprovecharse para su propio beneficio de la comercialización de este.

El problema con el que se enfrentaron fue que el bien necesario no posee ni las cualidades ni las características de otros bienes con los cuales se procedió de igual manera e.g. el agua, la tierra, los automóviles, la ropa, las casas, etc. Estos son bienes fisicos y como tales se los puede ver, palpar y hasta almacenar si es necesario. El

-Escuela de Ciencias de la Computación e Informática,Universidad de Costa Rica.

Department ofComputer and Information Science,University ofFlorida 
problema se da cuando un bien de carácter general se vuelve escurridizo e imposible de atrapar, cuando para producirlo basta prácticamente con una muestra. Consecuentemente, es imposible su comercialización por los medios tradicionales; máxime porque no se puede concentrar la producción en unas cuantas manos -pues la posibilidad de producirlo, aparentemente, está en toda persona y/o empresa. Una situación semejante se da en dos bienes parecidos: la música y los videos. Parecidos, pero a su vez como veremos totalmente distintos.

Por otro lado, al ser el bien de carácter general y eventualmente vital, una limitación. a su producción, venta o distribución puede tener nefastas consecuencias para el desarrollo de la humanidad, principalmente, para aquellos que están más necesitados. Se pretende ilustrar que, la nueva tecnología, ciencia o como se la quiera llamar es demasiado nueva como para ponerle restricciones o gobernarla con los enfoques industriales del siglo diecisiete; que cualquier paso erróneo puede tener consecuencias impredecibles para el desarrollo de la humanidad; que es mejor avanzar con pies de plomo que realizar propuestas de cambios legales precipitadas y unilaterales. Propuestas que de aprobarse únicamente pondrían en desventaja aquellos con menos visión del papel de la industria informática en el mundo.

\section{2.-LAS COPIAS PIRATAS DESOFTWARE, COPIAS DE IDEAS Y PERJUICIO CAUSADO.}

El objetivo, al comentar estos términos, es establecer cómo estos pueden ser fácilmente manipulados y alejados de su verdadero significado $\mathrm{y}$, por lo tanto, inducir a una concepción equivocada o incompleta del verdadero problema.

\section{1- Las copias "piratas" de software.}

Las siguientes definiciones para programa de computadora han sido dadas:

"Un programa de computadora es una serie de .instrucciones que le dice al computador qué hacer" . [Savage et al, 1986]
"El programa de computadora es un conjunto de afirmaciones o instrucciones para ser usadas directa o indirectamente en un ordenador con el fin de obtener un resultado determinado".

Ley de USA (96-517, Dic 1980) Derechos de Autor (t27 del US Code Act, art 101).

"El conjunto de instrucciones expresadas mediante palabras, códigos, planes o en cualquier forma que al ser incorporadas en un dispositivo de lectura automatizada, son capaces de que un ordenador ejecute determinada tarea $\mathrm{u}$ obtenga determinado resultado".

OMPI, Proyecto de Disposición Tipo para leyes en materia de autor.

Las dos últimas definiciones se tomaron de la recopilación sobre juristas y computadores hechas por J.D. Castro en [Castro, 1992].

Estas definiciones -aunque académicamente correctas- no muestran realmente el significado real de un producto de programación (software) en el amplio sentido de la palabra. De este modo, todo "conjunto de instrucciones" sin ninguna característica especial-salvo el ser "entendidas" por un computador- queda cubierto en la definición; dando lugar a que cualquier "programa" pueda considerarse como tal. Sin más preámbulo esta definición la aceptaron los organismos mencionados -con ligeras alteraciones o ampliaciones como afirmaciones, palabras, códigos y, al final" cualquier forma" - con el fin de incluir en las definiciones legales las diferentes fonnas o tipos de programas.

Particularmente, la ley USA sobre derechos de autor ha llevado a serios problemas legales en los cuales la definición de programa ha estado en juego [Hennessy, 1990 pp. 2431. En el caso citado por Hennessy y Patterson, $\mathrm{Nec}$ vs. Intel, dos jueces se han pronunciado aceptando microprogramas-series de instrucciones primitivas usadas para programar el control básico del procesador de una computadora- dentro de la definición dada en la ley. Esto decide -por el momento- la cuestión de si microprogramas pueden ser copiados para ser usados para otras computadoras. Asi, difícilmente alguien puede crear un computador con el mismo control básico impidiendo por lo tanto la expansión del mercado. Sin embargo, la cuestión no termina aquí, como Hennesy y Patterson lo dicen. 
Se puede decir que el producto de programación o software consta de cuatro partes vitales:

-La secuencia de Señales Magnéticas Almacenadas.

Se les denominará SEMA: señales electromagnéticas puestas en un medio (cintas, discos o disquettes) que las mantiene casi indefinidamente.

Las señales pueden ser interpretadas por un computador y proceder de acuerdo con ellas. Técnicamente se les llama "programa objeto".

- los programa-fuentes: son códigos nemotécnicos similar al lenguaje hablado y el cual correctamente procesado en una computadora puede generar el SEMA. Los programa-fuentes se diferencian, en general del SEMA, en que no pueden ser interpretados por el computador pero si pueden ser entendidos por expertos en computación. Existen casos particulares en donde los programafuentes y las Sernas son los mismos.

Los programa-fuentes se escriben en conocidos Lenguajes de computación.

- Los manuales describen cómo hacer uso correcto de las SEMA. Se dirigen al usuario y también al experto.

- El mantenimiento facilitado por el autor o por el proveedor de las SEMA para su alteración en caso de falla o necesidad de mejora de estas.

Como se puede ver las definiciones de programa de computadora dadas anterioqnente coinciden en parte- con las definiciones previas. Aceptando la primera definición, entonces un programa de computadora puede ser un programa objeto (las SEMA) o bien un programa-fuente. No se deben usar definiciones incompletas o ambiguas (definición del US Code) o difusa (definición de la OMPI) o posiblemente cambiantes con el tiempo cuando las nuevas máquinas puedan interpretar algoritmos -prácticamente- en lenguaje natural.

Desde este punto devista, la copia de un programa -en el sentido amplio de la palabra- solo se dará si los cuatro ingredientes necesarios están presentes en la copia.

Por otra parte, si a la copia se le atribuye el adjetivo pirata, implica, necesariamente, un acto de robo - con violencia, aunque esto, está intrínseco, en el acto de robar -. No se conoce un solo caso documentado de copia pirata en el sentido aqui mencionado.
Las llamadas "copias piratas" de software son a lo sumo copias -sin mediar violencia de algún tipo- de las SEMA y, en el mejor de los casos, con alguna fotocopia de los manuales. Sin embargo, dos partes importantes no se pueden copiar con tanta facilidad: los programa-fuentes $y$ el mantenimiento.

Por lo tanto, las "copias piratas"ni son copias ni son piratas.

En el mejor de los casos - pretendiendo reconocer una problemática al trasfondo- se puede hablar de hurto parcial de programas. Sin embargo, hurto implica despojar de un bien a su dueño, y cuando se copia no se está realizando un despojo. Se puede llamar por lo tanto copia parcial de programas, sin achacarle carácter delictivo -no existente o, al menos, no tipificado como tal- al acto en si.

Por lo tanto, se debe diferenciar entre los ténninos "copia de programas" , "copia de SEMAS o programas objeto" y "copia de programafuentes", para lograr dilucidar claramente el problema. Pero, definitivamente, usar el término "copia pirata" sin distinción es tergiversar y dar una versión equivocada de los hechos y conceptos relacionados.

2.2-¿Esla copia parcial de programas un daño para la sociedad?

Para concebir un acto como un delito debe existir dafio moral, material, personal o comercial a la persona fisica o jurídica.

Si bien es cierto, la copia parcial de programas deja la sensación de haber hecho algo incorrecto. Un gusanillo mueve la conciencia de los que conocen el esfuerzo que existe en el desarrollo de un producto de programación; no obstante, el conocimiento amplio -por una multitud de usuarios- de muchos productos de programación se debe a esa copia parcial.

Esto provocó un cambio de actitud de las casas, que al principio pusieron mil trabas para la copia de los SEMA. Eliminaron las trabas al considerar que era más el dafio que podían hacer a sus clientes que la eventual copia parcial de los programas. 
Esto además de la promoción indiscriminada de su producto -causado a suvez por la proliferación de copias parciales -aceleró su conocimiento y el advenimiento de más clientes- pues poco se puede hacer y garantizar con una copia parcial de los programas. El profesional, que se precia de serlo y que por lo tanto conoce las implicaciones de los cuatro factores antes mencionados, utilizará un producto completo para sus aplicaciones y no una copia parcial.

Por otra parte, muchos productos de programación son vendidos por los autores a las casas proveedoras. Son estas - muchas veces -las que reclaman los derechos patrimoniales cuando al autor se le pagó, a veces, apenas lo suficiente. Estas, negocian con el programa, sacando un provecho en muchos casos de 30 o más veces, siendo conservadores, del costo del producto.

Esta ganancia desmedida se considera especulación en muchos países y si bien se puede considerar legal, en algunas lati tudes, dista mucho de ser moralmente correcto. Más adelante se considerará esto con más detalle.

Aún así, queda un argumento extra del proveedor: dejó de percibir un posible ingreso al ser copiado parcialmente su producto. Entre las causas de las copias parciales podemos citar:

- No existe en el país un proveedor de programas con los cuatro ingredientes básicos.

- La copia parcial se debe al poco poder adquisitivo de quién la hace. Ya vimos su poca utilidad.

- El sobreprecio del producto completo hace prohibitiva su compra.

- La copia parcial se hace en muchos casos con fines personales sin ningún provecho comercial.

Desde este punto de vista, eliminar la copia parcial no ocasiona ni siquiera una venta parcial. Por lo tanto, el argumento de pérdida de ingresos potenciales se debilita.

Después de considerar estos hechos, cabe preguntarse, si realmente se daña al autor o casa proveedora cuando se realiza una copia parcial. Más bien, se puede argumentar lo contrario. Por tanto, la copia particular, sin fines de lucro y no comercial dificil mente constituiría o debería tipificarse como delito.

Sin embargo, existen casos clarísimos de suplantación de marcas y comercialización de programas con todos sus agravantes. Estos casos consisten en que detenninadas casas o personas distribuyen copias parciales -SEMA (programas objeto) y manuales copiados-pues el mantenimiento y los programa-fuentes no son tan fáciles de hurtar, copiar o adquirir, suplantando al autor original y cobrando beneficios por ello. Estos casos son ilegales -penados por la leyes de derecho de marca y falsedad comercial actualmente vigentes en todos los países.

En estos aspectos, la educación informática y el conocimiento de los factores que intervinien en un producto de programación, podría prevenir estas situaciones.

\section{3- Sobre la copia de ideas o métodos.}

En la discusión de la propiedad intelectual se habla, también, de la copia de ideas o métodos. Dos cortas aclaraciones son necesarias.

De hecho, existen copias de ideas o diseños de productos de programación oprogramas -softwarepero, del mismo modo que existen copias de ideas diseños para la fabricación de un producto, una casa, un carro, etc.

Se puede dictaminar sobre la copia de los programa-fuentes de la misma forma que los arquitectos pueden dictaminar sobre la posible copia de los planos obien, los quimicos dictaminan sobre la posible copia de fórmulas, etc.

Actualmente, ambos mercados existen y los conflictos se dirimen mediante las leyes y con el apoyo de las asociaciones o colegios profesionales.

\section{3.- LA SITUACIÓN TÉCNICA Y ECONÓMICA.}

Después de aclarar los conceptos anteriores, es necesaria una discusión sobre la situación tecnológica y económica. 


\section{1- La máquina de hacer copias.}

Para ilustrar este punto se expondrá, como ejemplo, la fabricación de automóviles. Utópicamente, supongamos que existe un máquina capaz de convertir cualquier material en un automóvilsemejante a cualquier modelo presentado como muestra.

Supongamosademás, quedado subajísimo costo, esta máquina está al alcance de todos y que el nuevo autoasíobtenidonoadmite reparacionesni cambios, pero funciona. La pregunta es ¿Cuánto vale el automóvil parecido que produce la máquina?. La respuesta es obvia: el costo del material utilizado pues el costo de la máquina se podría considerar despreciable.

Así, ¿Cuál sería la actitud de las empresas productorasdeMercedez-Bens o Cadillacs?.Dirían, que es ilegal la duplicación, que están perdiendo ventas y en último caso, que tienen derecho sobre los autos parecidos y que seles debe reconocer estos derechos. La duplicación será ilegal cuando se establezca como tal. Para garantizar cierto pago bastará que al registrar el auto parecido, se le cobre el respectivo derecho.

La venta no la están perdiendo pues el comprador no realizaría la compra. El auto es parecido, no igual, pues los planos, las instrucciones de emsamblaje y las nuevas versiones modificadas están en poder de la empresa fabricante- se pagará por el disefio y por los costos de fabricación del primer modelo o versión -los cuales son mínimos por su distribución entre todos los potenciales "compradores".

Este costo nunca llega a ser igual que el del primer modelo, ni siquiera el costo de los actuales pues las economías de escala no son comparables a la situación real. En otras palabras, producir un millón de autos parecidos tendría el costo de producirel primer modelo; si este cuesta un millón de dólares cada auto parecido se podría adquirir por un dólar.

\section{2-El enfrentamiento con una nueva tecnología y costo marginal cero.}

La utopía descrita anteriormente pondría en jaque la fabricación y, probablemente, solo quedarían fábricas especializadas en autos particulares. Se producirían nuevas versiones -pero no fabricando modelos en serie. El precio de cada uno de los autos sería ridículo y el beneficio lo obtendría la economía mundial. Lo perjudicial de esta situación sería mantener las fábricas de modelos en serie y sus precios, impidiendo la duplicación parecida y solicitando la compra en la fábrica -aunque la fábrica utilice la máquina "duplicadora"-.

Pues bien, si se cambia automóviles por programas y autos parecidos, por programas objeto o SEMAS; se tendrá una utopía hecha realidad.

Definitivamente, cada copia parcial de un programa tiene una cualidad económica única y nunca antes encontrada -excepto, tal vez, en la música y el video- y esta es su capacidad de reproducirse con un costomarginal prácticamente nulo (el costo del medio magnético) y por lo tanto, la capacidad de reproducirse indefinidamente $\sin$ dafio o perjuicio para alguien.

No obstante esta comparación, los programas requieren de mantenimiento y las nuevas versiones son mejores -situación inexistente en la música o el video, donde la primera versión es, muchas veces, suficiente.

\section{3- El precio del software.}

Por el análisis anterior, cabe preguntarse cuál es el precio de un producto de programación y cuál es el precio de un programa. La primera copia de un software - programa objeto, manuales, programa programa-fuente y costo del mantenimiento - tiene un costo determinado por la cantidad y calidad delos profesionalesy recursos implicados.

La copia de un programa objeto tiene, básicamente, el costo del medio en donde se copia y el tiempo profesional invertido. Todos estos costos debe cubrirlos quién ordena la primera copiay entonces seconvierteen dueño del software.

$\mathrm{Si}$ existe otro comprador potencial para el programa - en las mismas condiciones- se divide el costo entre los dos y así sucesivamente. Si las modificaciones al producto son necesarias para un tercer cliente, el costo para este será, básicamente, el costo marginal de esas modificaciones más una tercera parte del costo del producto original. 
Obviamente, la fijación de precios pocas veces se hace así y generalmente muchos compradores pagan el software como nuevo. De ahí, las ganancias tan exorbitantes potenciales -aun con una pequefta rebaja.

En la práctica, el autor debe estimar la demanda potencial-número de usuarios potenciales- y dividir el costo entre todos ellos.

La falta de un adecuado registro de quienes han adquirido -con fines comerciales- dicho programa y la potencial copia parcial; obliga al autor a fijar el precio al azar con el fin de recuperar su inversión. De cualquier forma, siempre se recupera la inversión pues la primera copia se vende a precio de costo o a un precio que asegure la recuperación rápida de esta; el resto es ganancia.

Es injusto que -recuperada con creces la inversión- el usuario pague como si fuera la primera copia o aun un precio relativamente alto, de acuerdo con la cantidad de copias vendidas. Una solución a esta problemática se logra si existe una política del autor a reintegrar parte del costo a los compradores anteriores conforme el producto se venda, Esto motivaria el registro de compradores e inhibiría la copia parcial pues quien la facilite estaría afectándose a sí mismo.

\section{4.- LOS PAÍSES DEL TERCER MUNDO SIN LUZ Y EL DERECHO DEL CONOCIMIENTO.}

"Conocimiento es poder y el computador una amplificación de ese poder"

E. Feigenbaum and P. Mc Corduck.

No cabe duda alguna de que el computador es un medio de acceso a la información y, también, a una forma más elaborada de esta, el conocimiento. El computador, originalmente, fue concebido como un equipo o hardware que se adquiere para solucionar un problema; sin considerar, en esta adquisición, programas más allá del sistema operativo. El computador se concibe como un todo integrado: equipo (hardware) y programas (software); donde este último adquiere cada vez mayor relevancia.

Dificilmente, se puede encontrar hoy un usuario que esté satisfecho con el equipo. Más bien, pasa aun segundo plano cuando se percibe la importancia de los productos de programación como parte vital e integral de un sistema de cómputo.

Así, la frase de Feigenbaum y Mc Corduck debería parafrasearse: "Conocimiento es poder y el programa de computadora una amplificación de ese poder". El conocimiento actual es vasto y extenso, procesarlo sin ayuda automatizada no tiene sentido. Limitar por lo tanto, el uso de los programas puede tener consecuencias nefastas para quien desee educarse y progresar. Los países del Tercer Mundo verían severamente limitada su capacidad de acceso al conocimiento básico y entonces las consecuencias para el desarrollo futuro impredecibles.

$\mathrm{Si}$ se toma en cuenta la relevancia de la informática en el mundo futuro, bastaría una simple analogía. Se la podría comparar en relevancia con la luz para el mundo actual y su descubrimiento en el siglo pasado. Si Tornas Alva Edison hubiera protegido su invención y exigido el pago de patentes onerosas -por la magnitud de su invención- entonces nadie podría imaginarse las consecuencias para el mundo actual. Probablemente encontrariamos al Tercer Mundo a obscuras y las capitales iluminadas con bombillas. Estas pueden ser las consecuencias para el futuro de la informática si se limitan, severamente las bases de su desarrollo. Acotar el acceso al conocimiento, mediante la limitación de programas, podría sumergir en el obscurantismo informático y cultural a los países tercemundistas

Negar los medios de acceso a la computación es negar el acceso al conocimiento.

El mundo actual tiene un ejemplo claro: Japón. Las leyes japonesas permitieron el desarrollo general del país gracias a la posibilidad de copia ya la adaptación de tecnología extranjera [Andre, 1993].

\section{5.- SOLUCIONES A LA PROBLEMÁTICA SOBRE PROTECCIÓN DE PROGRAMAS.}

Desde el punto de vista aqui presentado, se puede decir que permanecen las siguientes problemáticas: 
- Distribución comercial de copias parciales de productos de programación sin la adecuada autorización del autor; lesionando no solo a los autores sino también a los compradores de programas por la falta de respaldo sobre el producto ofrecido.

- Inadecuado registro de productos de programación. Actualmente se registran programas sin una definición clara o completa del producto a registrar.

- Falta de un organismo oficial cuya función sea dictaminar sobre la posible copia y el grado de dicha copia.

- Posible utilización de productos de programación para fines comerciales sin la adecuada adquisición de los mismos i.e., la utilización de un programa como un medio para la obtención de otro más elaborado.

- Falta de un registro global de licencias de productos de programación adquiridos -aunque esta adquisición haya sido solo parcial i.e., sin la adquisición de los programas programafuentes-o

La ausencia de este registro impide conocer cuáles son las copias de programas con derecho a circular y eventualmente, conocer las programa-fuente de una posible copia. Esta ausencia también impide conocer la cantidad de licencias vendidas de un producto $-\mathrm{y}$ por lo tanto conocer el precio adecuado para este- e impide el adecuado control sobre el uso intermedio de un producto de programación.

Estos problemas pueden ser evitados -o al menos minimizados- con las siguientes soluciones:

- El registro de marcas utilizado en cualquier país permite fácilmente detectar cuándo una persona física o jurídica está suplantando marcas. El procedimiento es similar para cualquier otro producto. De esta forma, se puede distribuir comercialmente un producto pero en ningún momento o circunstancia se puede mencionar o acreditar la marca de este sin la debida autorización. Si se hace subrepticiamente, el organismo oficial propuesto en las siguientes líneas permite dilucidar el asunto.

- Cambiar la definición de programa por productos de programación en los términos aquí mencionados y registrarlo de acuerdo con esto. Registrar programas -como simple secuencia de instrucciones para el computadores equivalente a registrar cualquier poesía sin serlo o cualquier panfleto como libro.

Obviamente, este registro incluiría todos los productos de programación realizados en el país o en venta.

- Tener una oficina de asesoría en el registro público cuyas funciones serían:

- Dictaminar casos de copia parcial o total y si se hizo distribución comercial.

- Rendir criterio sobre la "completitud" o integridad y calidad del producto de programación por registrar.

- Recibir denuncias sobre inadecuada distribución comercial y uso no autorizado de un producto con fines comerciales.

- Llevar un registro de las licencias de programas vendidos.

- Aconsejar o asesorar en disputas de precio de programas.

Esta oficina tendría el apoyo de la asociación o colegio de profesionales en informática y computación, el cual participaría como integrante de la dirección de sí misma y en la labor operativa de registro y dictámenes.

\section{6.- CONCLUSIONES.}

Lo mencionado anteriormente da las bases para una discusión seria y prometedora sobre lo que debe ser la protección jurídica de programas o productos de programación. Se espera haber arrojado luces sobre diferentes conceptos -mal utilizados con respecto a la problemática. Algunos 
conceptos expresados pueden lidiar con lo tradicional y con las diferentes legislaciones. Sin embargo, ha quedado claro el caracter innovador de la tecnologia informáticay suposible repercusión en el futuro. Los puntos aquí expuestos y las soluciones dadas distan de ser tan completas como el autor hubiera deseado, pero permiten crear una discusión en donde intervenga la comunidad informática y computacional sobre las repercusionesmorales delas legislacioneso tratados nacionales e internacionales propuestos. También se espera haber hecho clara la relación programaconocimiento y su relevancia para el desarrollo del Tercer Mundo. Se puede hablar, entonces, de un nuevo derecho humano y es el derecho al conocimiento, el derecho a educarse y formarse y el derecho al acceso del conocimiento sin el cual no puede desarrollarse.

Un agradecimiento a aquellos que con su escucha y paciencia permitieron refinar algunas de las ideas presentadas. A Villar Salvatierra por la revisión original del manuscrito, alos encargados de la revista por la corrección de innumerables faltas; no obstante todos las que permanecen son responsabilidad exclusiva del autor.

\section{7.- BIBLIOGRAFÍA}

[Andre, 1993) Andre M, 1993. Did USA sav ves to Japan. Internet post: monu6.cc.monash.edu.au pub/nihongo as Japanyes, 1993. Internet.

[Castro, 1992) Juan Diego CastroF. Juristas $\mathrm{V}$ Computadoras. Juriscomputación S.A. 1Ed. 1992. San José, Costa Rica.

[Hennessy, 1990) J. Hennessy \& D. Patterson. Computer Architecture. A quantitative approach. Morgan Kaufmann Publishers Inc. 1990.

[OMPI, 1990) OMPIy A1RIP. Simposiosobre propiedad Intelectual en América Latina. OMPI. Set. 1990. Usa.

[Salazar, 1992) Silvia Salazar \& otros. Primer Encuentro InterUniversitario de Propiedad Intelectual Conceptos básicos.. 1992. UCR 